Use of mirage effect for the detection of phase transitions in solids

This article has been downloaded from IOPscience. Please scroll down to see the full text article.

1993 Meas. Sci. Technol. 4435

(http://iopscience.iop.org/0957-0233/4/3/028)

View the table of contents for this issue, or go to the journal homepage for more

Download details:

IP Address: 117.211.83.202

The article was downloaded on 01/12/2011 at 09:49

Please note that terms and conditions apply. 


\title{
DESIGN NOTE
}

\section{Use of mirage effect for the detection of phase transitions in solids}

\author{
K Rajasree, V Vidyalal, P Radhakrishnan, V P N Nampoori \\ and $\mathbf{C} \mathbf{P}$ G Vallabhan \\ Physics Department, Cochin University of Science and Technology, Cochin 682022 , \\ Kerala, India
}

Received 22 October 1992, accepted for publication 2 November 1992

\begin{abstract}
The phenomenon of mirage effect suffered by a He-Ne laser beam has been utilized to detect phase transitions in solids. It has been observed that anomalous fluctuations of large amplitude occur in the signal level near the transition temperature. The mean square value of the fluctuation is found to exhibit a well-defined peak at this point. Results of measurements made in the case of crystals of TGS $\left[\left(\mathrm{NH}_{2} \mathrm{CH}_{2} \mathrm{COOH}\right)_{3} \cdot \mathrm{H}_{2} \mathrm{SO}_{4}\right]$ and a ceramic sample $\left(B a T i O_{3}\right)$ are given here to illustrate this technique.
\end{abstract}

The mirage technique which exploits the optical beam deflection due to a refractive index gradient was first introduced in the early 1980s (Boccara et al 1980). It is currently used as a very effective tool for optical spectroscopy, thermal measurements and imaging in solids. A hot body heats up the surrounding medium so as to generate a refractive index gradient (RIG) directed away from its surface. An optical beam (probe beam) propagating normal to the RIG and parallel to the hot surface suffers deflection from the original beam path (mirage effect). The amount of deflection is a function of the magnitude of the RIG in the vicinity of that surface and this in turn will depend on the various thermal parameters of the sample as well as the distance between the sample surface and the detector together with the relevant geometrical factors. The magnitude of the beam deflection can be measured using a position-sensitive detector (PSD).

To compute the thermal energy of a heated region by processing a detector signal, one has to correlate the temperature distribution of the investigated region with the optical beam propagation through the adjacent nonhomogeneous medium together with the detector response. Theoretical calculation of the probe beam deflection has been reported using a quadrant detector as the PSD (Jackson et al 1980). In the present investigation an optical fibre coupled to an avalanche photodiode (APD) acts as the position-sensitive detector. Such detectors have been employed for damage threshold determination using the photothermal deflection tech-

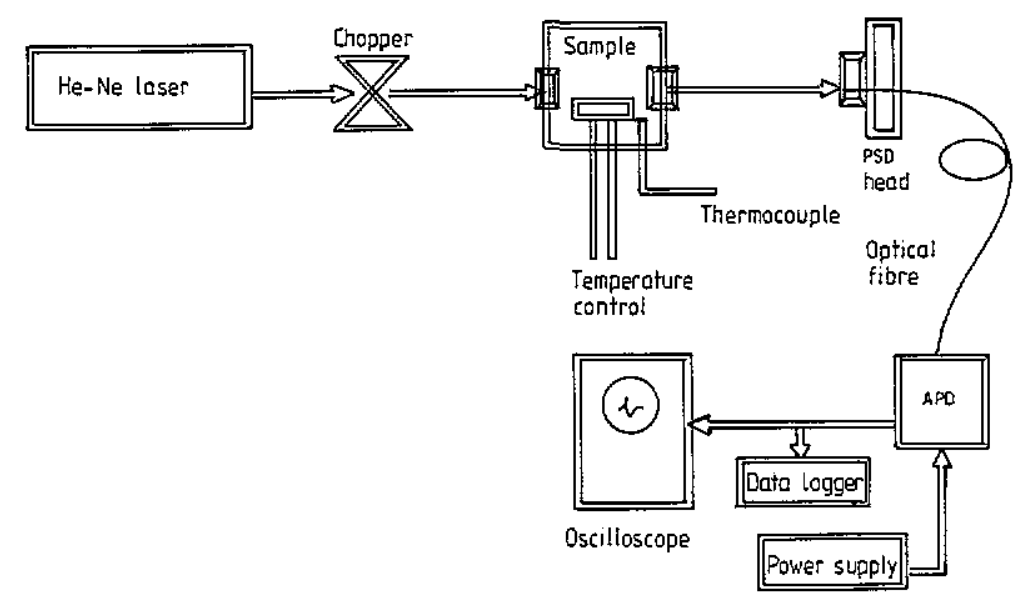

Figure 1. Schematic diagram of the experimental set-up. 
nique (PTD), for non-contact measurement of temperature and for flow velocity measurements (Rajasree et al 1992, 1991, 1990).

The schematic diagram of the experimental set-up is shown in figure 1. A chopped laser beam with a Gaussian cross section (FWHM $=0.84 \mathrm{~mm}$ ) from a stabilized $5 \mathrm{~mW}$ $\mathrm{He}-\mathrm{Ne}$ laser is made to pass, grazing the heated sample surface at a distance of $0.42 \mathrm{~mm}$. The sample is enclosed in a chamber with appropriate windows to avoid air convection which may cause erratic fluctuations of the probe beam. The specimen in the form of a rectangular slab or a circular disc (of about $1 \mathrm{~cm}$ diameter) is kept lapped to a copper block which is heated by an electrical heating element attached to a temperature controller. The PSD consists of a step-index multimode optical fibre with a core diameter of $80 \mu \mathrm{m}$ (cladding $120 \mu \mathrm{m}$ ) with the polished tip coupled to an avalanche photodiode (Thorn EMI Si APD type S30 500) at the other end. A specially designed highly stable, ripple-free variable voltage $(125-250 \mathrm{~V} \mathrm{DC})$ power supply is used to bias this detector so that it operates in the required unsaturated region of the operating characteristics of the APD. (The power supply uses electronic filtering, which provides a high ripple rejection ratio of $94 \mathrm{~dB}$.) The output of the APD is monitored across an appropriate load resistance using a digital AC voltmeter.

The fibre tip is mounted on an $X Y Z$ translator. Initially, at room temperature, the chopped probe beam is adjusted to fall on the polished tip of the fibre to get a maximum signal $\left(v_{0}\right)$ from the APD detector corresponding to the centre of the beam. As the sample surface is heated (at a rate of about $0.5^{\circ} \mathrm{C} \mathrm{min}-1$ ) the probe beam gets deflected and the PSD output $\left(v_{t}\right)$ is reduced. The difference $\left(v_{0}-v_{t}\right)$ is taken as the deflection signal. The temperature of the heated surface is measured using a thermocouple. As the rate of heating is small, the lag in the thermocouple reading is not appreciable for thin samples. A data logger is used to print the signal levels at intervals of $0.5 \mathrm{~s}$.

The above experimental set-up was used for the detection of phase transitions exhibited by solids. As the thermal properties of the sample undergo drastic variation at the phase transition temperature, phase transition is bound to affect the deflection signal in a measurable way due to the consequential changes produced in the RIG. Actually, near the transition temperature, wide fluctuations in the signal are observed. Figures $2(a)$ and $(b)$ display signal voltage as a function of temperature for two different kinds of sample, namely, TGS (triglycine sulphate: $\left(\mathrm{NH}_{2} \mathrm{CH}_{2} \mathrm{COOH}\right)_{3} \cdot \mathrm{H}_{2} \mathrm{SO}_{4}$ ) and a ceramic sample $\left(\mathrm{BaTiO}_{3}\right)$ respectively. The vertical bars in the plot indicate the extent of fluctuation in the signal. Sharp increases in signal fluctuation are observed at $49 \pm 0.5^{\circ} \mathrm{C}$ for TGS and at $125 \pm 0.5^{\circ} \mathrm{C}$ for $\mathrm{BaTiO}_{3}$. This anomalous behaviour in the signal obviously arises due to rapid changes in the thermal parameters in the material during a phase transition. Different regions of the sample undergo phase transitions at slightly different instants and this in turn yields fluctuations in the overall values of thermal parameters of the sample. Such a
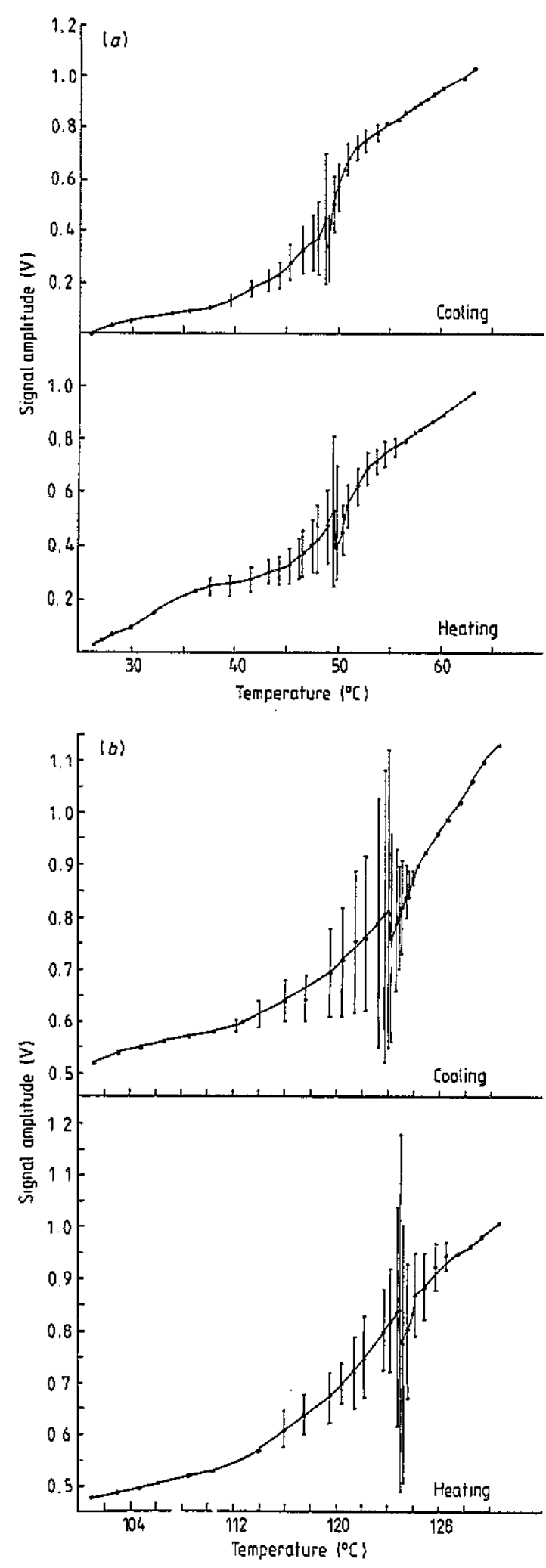

Figure 2. (a) The plot of the signal versus temperature for TGS. (b) The plot of the signal versus temperature for $\mathrm{BaTiO}_{3}$.

process will result in temperature fluctuations of the sample surface which affect the RIG in the adjacent gas (air) medium. A similar phenomenon has been observed in the case of light scattering from solids near critical temperature (Fleury and Lyons 1983). Solid samples 
which do not exhibit any phase transition did not exhibit any fluctuation in the signal amplitude in this temperature region. Figure $3(a)$ and $(b)$ show the plot of mean square deviation of the signal against temperature for TGS and $\mathrm{BaTiO}_{3}$ respectively. The mean square values of the signal fluctuations exhibit a clear peak near the phase transition temperature. The hysteresis phenom. enon, which is typical for most ferroelectric crystals, is also observed on cooling the heated sample. The transition temperatures of the TGS crystal and $\mathrm{BaTiO}_{3}$ measured using the present technique are found to agree with those reported earlier (Seo et al 1990, Kittel 1971).

In the present experimental set-up, apart from signal fluctuations due to sample properties above ambient temperature, thermal fluctuations due to Bernard convection type instability (Haken 1978) may also induce similar fluctuations. However, the latter type of fluctuations will appear as a background to the largeamplitude fluctuations arising due to phase transitions. This is clear from figures $2(a)$ and (b) where above and
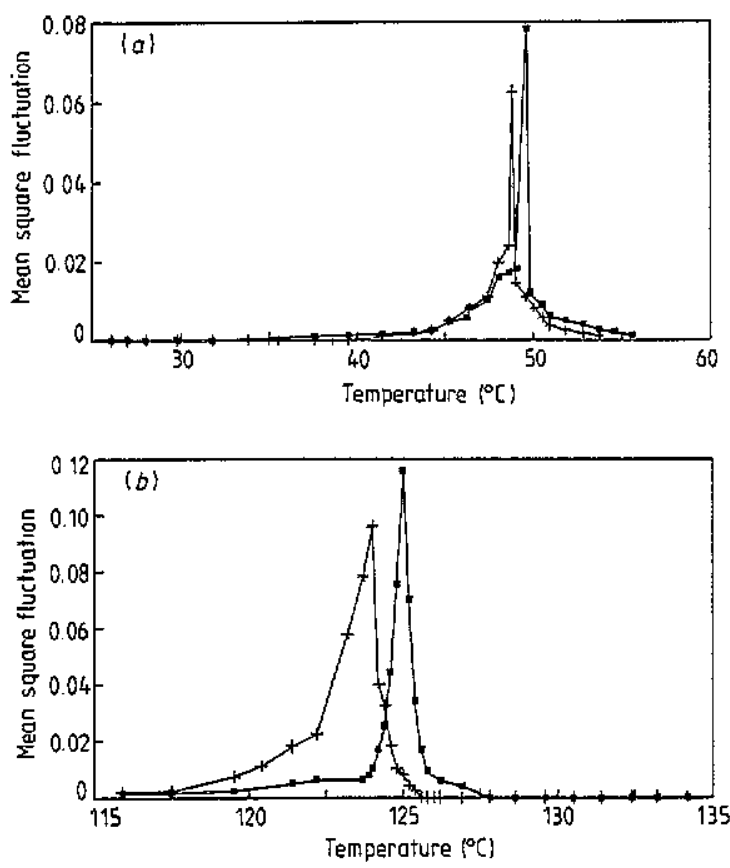

Figure 3. (a) Plot of mean square deviation of the signal with temperature for TGS. (b) Plot of mean square deviation of the signal with temperature for $\mathrm{BaTiO}_{3}$. Symbols denote heating ( $\boldsymbol{U})$ and cooling (+). below the phase transition temperature fluctuations are very small in magnitude. However, when one wants to build up an exhaustive theory of PTD due to phase transitions, Bernard convection should also be taken into consideration. The validity of the present results will, nevertheless, remain unaffected by such convectiontype instabilities generated over a heated sample surface.

The technique described here is much simpler than direct measurements of temperature dependences of dielectric constant, index of refraction or $\partial n / \partial T$ and offers a very good experimental tool for studying phase transitions in materials, including superconducting samples. The present technique also avoids the use of a pump beam which requires a more complex set-up (Seo et al 1990). In conclusion, we have demonstrated in this note that mean square signal fluctuations of the mirage effect are a very good indicator of the occurrence of phase transitions in materials.

\section{Acknowledgments}

KR and VV wish to thank the UGC and the CSIR, New Delhi, for their research fellowships.

\section{References}

Boccara A C, Fournier D and Badoz J 1980 Thermo-optical spectroscopy; detection by mirage effect Appl. Phys. Lett. 36 130-2

Fleury P A and Lyons K B 1983 Light Scattering Near Phase Transitions ed $\mathrm{H} Z$ Cumming and Levanyuk (Amsterdam: North-Holland) pp 45I-99

Haken H 1978 Synergetics: An Introduction, Non Equilibrium Phase Transition and Self Organization in Physics, Chemistry and Biology 2nd edn (Berlin: Springer) pp 7, 245

Kittel C 1971 Introduction to Solid State Physics 4th edn (New York: Wiley) pp 476

Jackson W B, Amer N M, Boccara A C and Fournier D 1980 Photothermal deflection spectroscopy and detection Appl. Opt. 20 1333-44

Rajasree K, Ravikumar A V, Radhakrishnan P, Nampoori V P N and Vallabhan C P G 1992 Damage threshold determination of bulk polymer samples using pulsed photothermal deflection technique Bull. Mater. Sci. 15 183-8

Rajasree K, Vidyalal V, Radhakrishnan P, Nampoori V P N and Vallabhan C P G 1991 to be published

Rajasree K, Ravikumar A V, Radhakrishnan P, Nampoori V P N and Vallabhan C P G 1990 Detection of air flow from laser irradiated surface using pulsed photothermal deflection technique $J$. Acoust. Soc. India 18 24-7

Seo H J, Yun S I, Min Su Jang and Kim T Y 1990 Phase transition of GMO and TGS crystals studied by a photothermal beam-deflection technique (Springer Series in Optical Sciences 62) (Berlin: Springer) pp 303-5 